\title{
Hochschulmanagement - Auf dem Weg zu einer neuen Profession?
}

\begin{abstract}
Ob Internationalisierung, Berufsberatung oder Qualitätssicherung-Beispiele für gewachsene Erwartungen an und neue Serviceaufgaben für das administrative Hochschulmanagement gibt es viele. Dabei lässt sich seit einigen Jahren auch die Etablierung neuer Tätigkeitsbereiche beobachten, die oftmals zwischen Wissenschaft und Verwaltung angesiedelt sind. Bislang ist jedoch wenig bekannt über das Berufsfeld „administratives Hochschulmanagement“. Auf der Basis von Ergebnissen einer deutschlandweiten Fragebogenerhebung, Interviews und der Auswertung von Stellenanzeigen zeichnet der Beitrag ein genaueres Bild dieser Tätigkeitsbereiche und zeigt ihre zentralen Charakteristika aus organisations- und professionstheoretischer Sicht auf. ${ }^{1}$
\end{abstract}

\section{Einleitung}

Reformen im Hochschul- und Wissenschaftssektor bedeuten auch Veränderungen für die Struktur, Arbeitsweise sowie Tätigkeitsfelder im Bereich der administrativen Organisationsbereiche an deutschen Hochschulen. Parallel zum Outsourcing einzelner Arbeitsbereiche lässt sich der Aufbau neuer Organisationseinheiten und Stellen wie z. B. für Qualitätsmanagement, Forschungsmanagement, Technologie-Transfer und $\mathrm{Ca}$ reer Service beobachten (Adamczak et al. 2007; Luckwald, von 2008). Die mit der Übernahme zahlreicher neuer Planungsund Servicefunktionen verbundene Einrichtung neuer Stabsstellen und Projektbereiche an vielen Hochschulen führt $\mathrm{zu}$ einer stärkeren Ausdifferenzierung der herkömmlichen Dezernatsstruktur der Hochschulverwaltungen. Darüber hinaus ist es zur Etablierung neuer Expertengruppen oder sogenannter „neuer Hochschulprofessionen“ gekommen, die in ihrer Funktion und Tätigkeit zumeist zwischen klassischer Routineverwaltung und Wissenschaft angesiedelt sind (Klumpp/Teichler 2005, S. 2). Damit scheinen sich in den letzten Jahren neue Berufsbilder entwickelt zu haben, die wir im Folgenden als „administratives Hochschulmanagement" bezeichnen.

Doch was bedeutet die Übernahme neuer Managementstrukturen und -funktionen für die Hochschule und was sind zentrale Charakteristika des Tätigkeits- profils, des beruflichen Werdegangs und Selbstverständnisses der im , administrativen Hochschulmanagement" Tätigen? Inwiefern kann von der Entwicklung einer neuen Profession in diesem Bereich gesprochen werden?

Zur Beantwortung dieser Fragen skizzieren wir zunächst in Abschnitt 2 zentrale Besonderheiten und aktuelle Veränderungen in der Hochschulorganisation, da sich nur so spezifische Erfordernisse des Berufsfeldes Hochschulmanagement verstehen lassen. Es wird argumentiert, dass sich organisatorische und berufliche Veränderungen im Bereich des administrativen Hochschulmanagements vor dem Hintergrund einer grundlegenden Transformation von Hochschul- und Wissenschaftsorganisationen vollziehen. An die Stelle loser gekoppelter Systeme treten zunehmend strategiefähige Akteure, die über die entsprechenden Handlungskapazitäten verfügen müssen. Abschnitt 3 erörtert auf der Basis von Ergebnissen eines Forschungsprojekts aktuelle Entwicklungen und zentrale Charakteristika des Berufsfeldes administratives Hochschulmanagement. Abschnitt 4 interpretiert und diskutiert die Befunde. Dabei zeigt sich, dass der Werdegang der in diesem Bereich Tätigen durch heterogene Studienhintergründe und eine starke Affinität zum Hochschulund Wissenschaftssektor gekennzeichnet ist. Zudem verfügen Mitarbeiter über eine starke Identifikation mit der Hochschulorganisation und ein eher administrativunterstützendes Selbstverständnis. Trotz einer sich schnell entwickelnden Einbettung in berufliche Netzwerke sehen wir gegenwärtig jedoch nur wenig Anzeichen für die Herausbildung eines eigenständigen Berufsbildes oder einer Profession „Hochschulmanager bzw. Hochschulmanagerin“.

\footnotetext{
1 Der Beitrag basiert auf Ergebnissen eines Forschungsprojektes am Deutschen Forschungsinstitut für öffentliche Verwaltung Speyer 2007-2009 www.http://www.foev-speyer.de/hochschulprofessionalisierung/inhalte/01_home.asp. Neben einer Inhaltsanalyse von Stellenausschreibungen in "Die Zeit" von 1996-2007 wurden LeitfadenInterviews mit 27 Mitarbeiterinnen und Mitarbeitern im administrativen Hochschulmanagement sowie deutschlandweite Befragungen mit Kanzlern und Kanzlerinnen $(\mathrm{N}=153)$ sowie leitenden Mitarbeitern und Mitarbeiterinnen unterschiedlicher Funktionalbereiche durchgeführt $(\mathrm{N}=492)$.
}

Georg Krücken, Prof. Dr. rer. soc.,
Stiftungslehrstuhl für Wissenschafts-
organisation, Hochschul- und Wissenschafts
management an der Deutschen Hochschule
für Verwaltungswissenschaften (DHV)
Speyer. Arbeitsschwerpunkte: Wissenschafts-
und Hochschulforschung, Organisations-
soziologie.
e-mail:kruecken@dhv-speyer.de
Albrecht Blümel, Dipl.-Pol., ist wissenschaft-
licher Mitarbeiter am Stiftungslehrstuhl
Wissenschaftsorganisation an der DHV
Speyer. Arbeitsschwerpunkte: Organisations-
und Berufsforschung, Management in Non-
Profit- und Wissenschaftsorganisationen
e-mail: bluemel@dhv-speyer.de
Katharina Kloke, Dipl.-Soz., ist
Forschungsreferentin am Deutschen
Forschungsinstitut für Öffentliche
Verwaltung Speyer. Arbeitsschwerpunkte:
Organisations- und Professionssoziologie,
Wissenschafts- und Hochschulforschung
e-mail: kloke@foev-speyer.de




\section{Rahmenbedingungen des Hochschulmanagements}

\subsection{TRANSFORMATION DER HOCH- SCHULE ALS "ORGANISATIONALER AKTEUR}

Unserer Einschätzung nach befinden wir uns gegenwärtig in einer Phase, in der sich die Rahmenbedingungen des Hochschulmanagements deutlich verändern, auch wenn diese Veränderungen nicht immer bruchlos in modifizierte organisationale Praktiken umgesetzt werden. Trotz dieser Einschränkungen kann jedoch konstatiert werden, dass ein grundlegender Veränderungsprozess stattfindet, der darin besteht, dass Hochschulen zunehmend in handlungs- und entscheidungsfähige Akteure transformiert werden, die sich eigene Ziele setzen, Strategien verfolgen und die dafür erforderlichen Strukturen aufbauen. ${ }^{2}$ Diese Entwicklung ist keineswegs auf Deutschland beschränkt, sondern lässt sich in ganz unterschiedlichen nationalen Hochschulsystemen beobachten.

Im Anschluss an Krücken/Meier (2006) lassen sich dabei vier, sich wechselseitig unterstützende Elemente voneinander unterscheiden:

(1) Die allgemeine Rechenschaftspflicht der Organisation nimmt zu. So werden Universitäten durch formale Qualitätskontrollen, Hochschulrankings und -evaluationen sowie Zielvereinbarungen zunehmend als Gesamtorganisation adressiert.

(2) Es werden hierarchische Entscheidungsstrukturen innerhalb der Universität geschaffen. Eine jüngere Analyse zeigt, dass in allen 16 deutschen Landeshochschulgesetzen die Stellung der Rektoren und der Dekane gestärkt wird und diese mit neuartigen Machtbefugnissen ausgestattet werden (Hüther 2009). ${ }^{3}$ Diese Entwicklung ist in einen internationalen Trend eingebettet. Generell gewinnt „leadership" in Hochschulen an Bedeutung, wie Burton Clark $(1998,2004)$ in verschiedenen Fallstudien zu universitären Transformationsprozessen in Europa, Amerika und Afrika gezeigt hat.

(3) Es werden spezifische Leitbilder und Organisationsprofile definiert. Während die „Idee der Universität“ (Schelsky 1963) genau darauf basiert, dass man die Universität als Institution versteht, d.h. als unhinterfragtes Konzept, das hinreichend diffus und unbestimmt bleiben muss, um als allgemeine Institution anerkannt $\mathrm{zu}$ werden, finden gegenwärtig individuelle Profilbildungsprozesse statt, die die Universität als Organisation schärfer konturieren. Die Selbstpositionierung von Universitäten als Organisationen im Wettbewerb - wo möchte man im Vergleich zu anderen stehen? - impliziert die Kreierung von Leitbildern und Profilen sowie die Benennung hierauf bezogener Organisationsziele. Die Universität als Institution tritt demgegenüber in den Hintergrund, auch wenn in der empirischen Analyse Leitbilder und Profile häufig eine deutliche Nähe zu traditionellen Annahmen der Universität als Institution aufweisen (Kosmützky 2010).

(4) Und schließlich führt die zunehmende Verfasstheit der Universität als eigenständiger und handlungsfähiger Organisationsakteur zu einer fortschreitenden Differenzierung und Spezialisierung der Hochschulverwaltung, da die Universität ihre organisatorische Zuständigkeit in immer mehr Handlungsbereiche ausdehnt - vom Technologietransfer über die Frauengleichstellung bis zur Personalentwicklung. Die Oxford University beispielsweise verfügte 1920 nur über eine eigenständige Verwaltungseinheit, nämlich die Buchhaltung, während der Bereich „University Administration and Services" im Jahr 200916 Einheiten umfasste, die wiederum zum Teil intern differenziert sind.

\subsection{ORGANISATIONSWANDEL UND STÄRKUNG DES INSTITUTIONELLEN MANAGEMENTS}

Auch wenn Universitäten mit zu den ältesten bekannten Organisationen gehören, die gegenwärtig existieren, ist die Stärkung der Organisationsebene in Wissenschafts- und Hochschuleinrichtungen alles andere als trivial. Im Unterschied zu Wirtschaftsorganisationen werden Universitäten in der einschlägigen Forschung als vergleichsweise führungs- und strukturschwache Organisationen beschrieben, die gegenüber ihren professoralen Mitgliedern, den Disziplinen und dem Staat nur über geringe Handlungs- und Entscheidungskompetenzen verfügen.
So spricht Mintzberg (1979) von Universitäten als dem Prototyp der „professionellen Bürokratie“, d.h. einer von wissenschaftlichen Experten geleiteten Organisation, in der der Rektor bzw. die Rektorin oder der Präsident/die Präsidentin über keine spezifischen Managementkenntnisse verfügt und in erster Linie Wissenschaftler ist. Gegenüber seinen Peers, den anderen Professoren und Professorinnen, fungiert er oder sie als Primus inter pares, nicht als Vorgesetzter, dessen Aufgabe die Durchsetzung von Organisationszielen ist. Parallel dazu ist es Aufgabe der dem Kanzler oder der Kanzlerin unterstellten Hochschuladministration, die Rechtskonformität des Entscheidens zu sichern und für den möglichst reibungsfreien Ablauf der innerorganisatorischen Handlungsabläufe zu sorgen.

Dieses Modell der Hochschulorganisation weist, was nicht überrascht, angesichts der langen Universitätsgeschichte und der Besonderheiten der Organisation wissenschaftlicher Arbeit erhebliches Beharrungsvermögen auf. Jedoch gerät es zunehmend unter Druck. So haben weitgreifende Veränderungen der GovernanceStrukturen seit Mitte der 1980er Jahre nicht nur in Deutschland, sondern in allen europäischen Hochschulsystemen zu einem Rückzug des Staates aus der Detailsteuerung von Hochschulen und zu einer Stärkung des institutionellen Hochschulmanagements geführt (Lange/Schimank 2007). Zudem bedeutet die Einführung betriebswirtschaftlicher Steuerungsinstrumente an deutschen Hochschulen, wie z.B. Kontraktsteuerung über Ziel- und Leistungsvereinbarungen, Output-orientierte Budgetierung sowie Qualitätsmanagementsysteme für Forschung und Lehre, auch tief greifende Reorganisationsprozesse (Ziegele 2005; Nickel 2007)

2 Obwohl im Folgenden von Hochschulen gesprochen wird, geht es hier jedoch zumeist um Universitäten, da sich die hier skizzierten Veränderungen vor allem auf einen Typus von Hochschulen konzentrieren, der formal über das Lehrstuhlprinzip und die Differenzierung nach wissenschaftlichen Disziplinen, die Ausrichtung auf Forschung und Lehre sowie das Selbstverständnis als einer globalen Institution definiert ist.

3 Die Analyse von Hüther (2009) zeigt aber auch sehr deutlich die Grenzen dieser Machtbefugnisse, da Rektoren und Dekane bei Professoren nach wie vor weder die Möglichkeit haben, über Aufstiegsund Karrierewege zu disponieren, noch über die in anderen Organisationen zur Verfügung stehenden Sanktionsmöglichkeiten (Versetzung, Kündigung) verfügen. 
innerhalb der Hochschulen. Diese Entwicklungen sind nicht unumstritten. Kritiker befürchten, dass in Universitäten und Wissenschaft monetäre Aspekte die Oberhand gewinnen. Hochschulmanagement gilt aus dieser Perspektive als ausgesprochen problematisch, gar als wissenschaftsfremd.

\subsection{ANFORDERUNGEN UND GRENZEN DES HOCHSCHUL- MANAGEMENTS}

Historisch betrachtet ist Management jedoch vor allem eine Antwort auf die gestiegene Komplexität in Organisationen und Gesellschaft. Wenn im Hochschulmanagement als Bezugspunkt „Komplexität“ und nicht „Geld“ gewählt wird, kann man das Hochschulmanagement weder als Allheilmittel für knappe Ressourcen betrachten noch als Mittel für eine schleichende Entmachtung von Wissenschaftlerinnen und Wissenschaftlern, die unter den Druck einer nicht mehr wissenschaftlichen, sondern betriebswirtschaftlichen Rationalität gestellt werden. Die Notwendigkeit des Hochschulmanagements entwickelt sich somit vielmehr aus der grundlegenden Dynamik des Hochschul- und Wissenschaftssystems selbst und seiner gesellschaftlichen Umwelten heraus.

So besteht eine interessante Parallele zur Entwicklung von Managementfunktionen und -rollen des Managements in Industrieunternehmen, die im späten 19. und frühen 20. Jahrhundert einsetzten (Chandler 1977). Ursache hierfür war das Größen- und Komplexitätswachstum in den Unternehmen selbst sowie in den für das Unternehmen relevanten Umwelten. Beides entzog den Eigentümern zunehmend die Grundlage, ihre Unternehmen und die dort Arbeitenden selbst zu führen. Aus Familienunternehmen wurden von Managern geführte Unternehmen, und die Trennung zwischen Eigentum und Führung setzte sich durch.

Auch Hochschul- und Wissenschaftsorganisationen und ihre Umwelten sind durch eine erhebliche Komplexitätssteigerung gekennzeichnet. Die Zunahme neuer Aufgaben - von der interdisziplinären Kooperation über die Studierendenauswahl bis zur Qualitätskontrolle - bedeutet eine deutliche Steigerung der Systemkomplexität, die eng mit der Komplexitätssteigerung in der Umwelt von Wissenschaftsorganisationen zusammenhängt.
Es ist jedoch davon auszugehen, dass es - im Unterschied zu Wirtschaftsorganisationen - in Wissenschaftsorganisationen Grenzen gibt, die neuen Aufgaben auf spezialisierte Managerinnen und Manager zu übertragen. Eine Delegation der Kontrolle über die grundlegenden Arbeitsprozesse in der Wissenschaft - Lehre und Forschung - an andere, nämlich Managementspezialisten, ist in der Wissenschaft nicht in dem Maße wie in der Wirtschaft möglich. Insofern in Hochschulorganisationen das Management kaum Möglichkeiten hat, sich des Engagements der Wissenschaftlerinnen und Wissenschaftler durch die Beeinflussung von Karriere- und Aufstiegschancen zu versichern, wäre eine Verlagerung von Kompetenzen auf spezialisierte Managerinnen und Manager schon aus organisationsstrukturellen Gründen zum Scheitern verurteilt. Reputation und die damit verbundenen Karrierechancen werden im Wissenschaftssystem typischerweise organisationsextern, d.h. durch die scientific community, zugeteilt. Durch die Organisation vermittelte Anreize und Sanktionen sind bei verbeamteten Professorinnen und Professoren, die sich zudem in der Regel durch eine starke organisationsexterne Orientierung auszeichnen, kaum als Steuerungsinstrumente des Managements einsetzbar. Dasselbe gilt für den wissenschaftlichen Nachwuchs, da erfolgreiche Nachwuchswissenschaftlerinnen und -wissenschaftler bis auf die wenigen Fälle von Tenure-Track-Optionen ${ }^{4}$ gezwungen sind, die Organisation zu verlassen.

Die Delegation der Kontrolle über die Arbeitsprozesse Lehre und Forschung ist aber auch aus sachlogischen Gründen nicht in dem Maße möglich wie in einfachen industriellen Fertigungsprozessen. Dies gilt einerseits für Prozesse der akademischen Lehre, da sich Lehr- und Lernprozesse kaum in klaren Ursache-Wirkungs-Ketten ausdrücken und beherrschen lassen. Vielmehr dominieren situative und personenbezogene Spezifika. Dasselbe gilt für die Forschungstätigkeit. Der britische Chemiker und Wissenschaftsforscher Michael Polanyi hat in diesem Zusammenhang bereits 1966 den Begriff des „tacit knowledge“, des stillschweigenden und impliziten Wissens, geprägt (Polanyi 1966). Dieses Wissen ist stark personen- und situationsbezogen und lässt sich nur in der konkreten Praxis des Forschungshandelns erschließen. Das Wissenschaftssystem ist, trotz seiner formalen Kommunikationsstruktur, sehr stark an konkrete Personen und Interaktionssituationen gebunden. Wissen in Universitäten, das sich auf die Kernprozesse Forschung und Lehre bezieht, kann also auch aus sachlogischen Gründen nicht umfassend an spezialisierte Managementexpertinnen und -experten delegiert werden, die Forschung und Lehre von außen steuern. Dies gilt selbst für nicht zum engeren Kernbereich gehörende Tätigkeiten wie etwa Technologietransfer (Krücken 2003).

Nimmt man den hier nur stichwortartig skizzierten Zusammenhang von Komplexitätssteigerung und Organisationsentwicklung in der Wissenschaft ernst, so bedeutet dies für die Etablierung des Hochschulmanagements zweierlei: Erstens macht es weiterhin durchaus Sinn, mit internen Systemkenntnissen und den spezifischen Arbeitsprozessen vertraute Wissenschaftler als Dekane oder Rektoren zu beauftragen, und nicht externe Manager aus dem Wirtschaftssystem. Zugleich sind jedoch zweitens im Zuge der zu Beginn skizzierten Transformation der Universität in einen eigenständigen, handlungs- und entscheidungsfähigen Akteur eine Stärkung der Organisationsspitze und ein damit einhergehendes Rollenverständnis erforderlich. Letzteres hat zwar den Besonderheiten der Organisation von Wissenschaft Rechnung zu tragen, bedeutet aber einen Identitätswandel, da man nun nicht mehr Primus inter pares ist, sondern eine Leitungsfigur mit individueller Verantwortung für die Gesamtorganisation und ihre Ziele.

Dieser Rollen- und Identitätswandel erfordert grundlegende Kompetenzen im Wissenschaftsmanagement und bleibt nicht nur auf die sogenannte Leitungsebene beschränkt. Er zeigt sich auch bei anderen, zum Teil bereits schon auf der Ebene des wissenschaftlichen Nachwuchses. So sind junge Wissenschaftlerinnen und Wissenschaftler, die etwa im Rahmen von Exzellenzprogrammen eine Forschergruppe leiten, mit einer Vielzahl, der Komplexität der Wissenschaftsorganisation und ihrer Umwelten geschuldeten Herausforderungen etwa in den Bereichen „Personal“, „Qualität“ und „Kommunikation“-konfrontiert, die Wissenschaftsmanagement im Interesse der eigenen Arbeit erforderlich machen.

\footnotetext{
4 Tenure-Track-Optionen stellen Nachwuchswissenschaftlern einen kalkulierbaren Karriereweg an der sie einstellenden Hochschule in Aussicht. Vgl. dazu ausführlich Sondermann et al. in diesem Heft.
} 


\section{Abb. 1: Etablierung neuer Stellen und organisatorischer Einheiten im Hochschulbereich}

Frage: In welchen Bereichen wurden an Ihrer Hochschule in den letzten 5 bzw. 10 Jahren neue organisatorische Einheiten/Stellen geschaffen? (\% der Ja-Antworten)

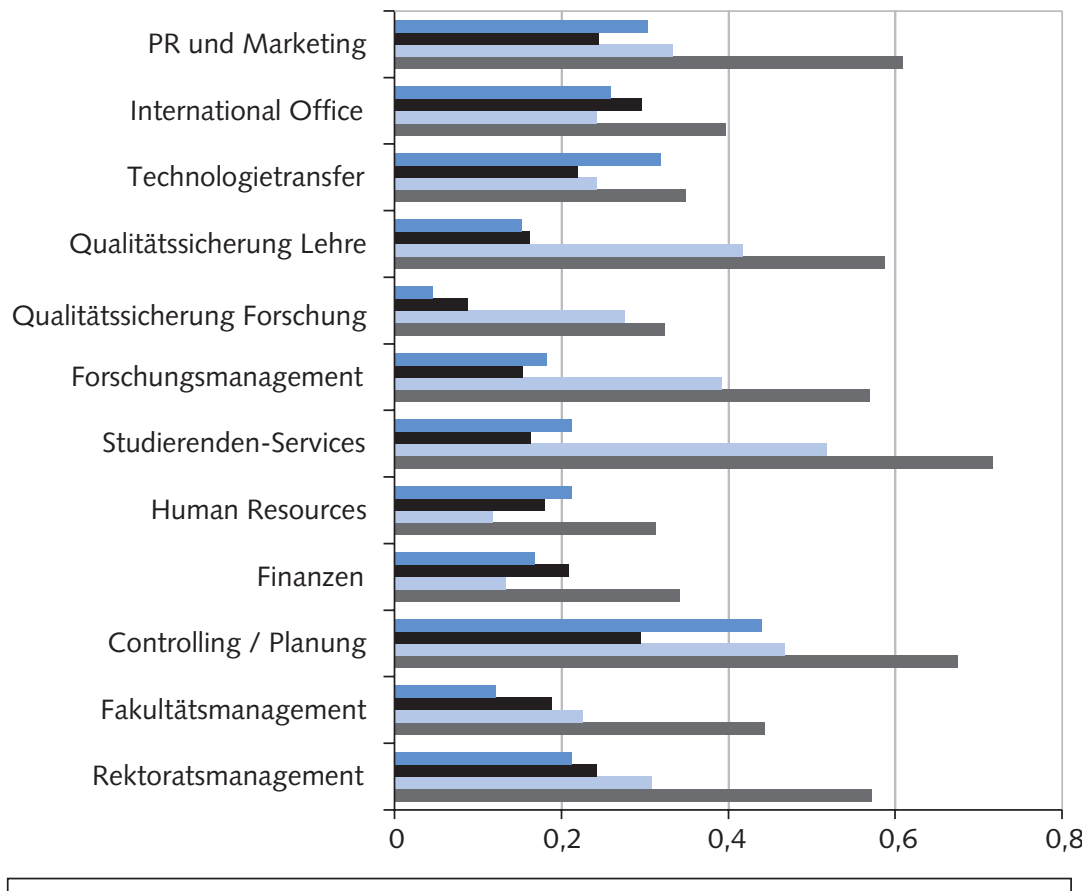

0-5 Jahre Stellen 0-5 Jahre Einheiten $\mathbf{0 - 1 0}$ Jahre Stellen $\mathbf{0 - 1 0}$ Jahre Einheiten

Komplementär zur Veränderung der Rollenverständnisse im Wissenschaftsbereich, und zwar sowohl auf der Leitungsebene (Rektoren, Dekane) als auch auf der Ebene des wissenschaftlichen Nachwuchses, ist seit einigen Jahren auch die Herausbildung neuer Positionen, Berufsbilder und Karrierewege im Bereich des administrativen Hochschulmanagements zu beobachten.

\section{Entwicklung und Charak- teristika des Berufsfelds "administratives Hoch- schulmanagement"}

Die skizzierte Transformation der Universität in einen handlungs- und entscheidungsfähigen Akteur bedeutet ebenfalls den Aufbau entsprechender administrativer Handlungskompetenzen. Grundlegend kommt es zu einer zunehmenden Ausdifferenzierung der Tätigkeitsfelder und Organisationsstrukturen innerhalb der herkömmlichen Hochschulverwaltung (Krücken et al. 2009). So implizieren die Umsetzung neuer Steuerungsinstrumente, wie z. B. Forschungsevaluation und Qualitätsmanagement, sowie der Auf- und Ausbau serviceorientierter Angebote wie Studien- und Berufsberatung, Alumni-Arbeit, interne und externe Wissenschaftskommunikation und Technologietransfer, auch die Entwicklung neuer, mitunter spezialisierter Organisationseinheiten und administrativer Supportstrukturen. Darüber hinaus ist an den meisten Hochschulen auch die Etablierung neuer Stellen am Schnittpunkt zwischen Hochschuladministration und Wissenschaft zu beobachten. Beispiele hierfür sind Fakultätsreferenten, Forschungsreferenten, Koordinatoren von Sonderforschungsbereichen, Exzellenzclustern oder Graduiertenschulen.

Dass es insbesondere in den letzten fünf bis zehn Jahren zu einem Reorganisationsprozess der Hochschulverwaltungen und einem deutlichen Anstieg an Stellen in neuen, spezialisierten Organisationseinheiten im Hochschulmanagement gekommen ist, machen die Antworten von Kanzlerinnen und Kanzlern bzw. hauptamtlichen Vizepräsidenteninnen und Vizepräsidenten an deutschen Hochschulen deutlich. Sie wurden im Rahmen einer deutschlandweiten Umfrage gefragt, in welchen Bereichen neue Organisationseinheiten und Personalstellen in den letzten fünf oder zehn Jahren geschaffen oder substanziell reorganisiert wurden. Das Ergebnis zeigt, dass zum einen vor allem in Bereichen Stellen und/oder Organisationseinheiten geschaffen wurden, die eine hohe Relevanz für die Legitimation und Kommunikation der Hochschule gegenüber externen Stakeholdern haben. So geben mehr als $60 \%$ der Kanzler an, in den Bereichen PR und Marketing vor allem in den letzten fünf Jahren sowohl neue Stellen geschaffen als auch neue Organisationseinheiten eingerichtet zu haben (Abbildung 1). Zum anderen wurden ebenso häufig Stellen oder organisatorische Einheiten etabliert, die zentral für die interne Steuerung durch die Hochschulleitung oder die Fakultätsleitung sind und dementsprechend auf die zunehmende Entwicklung der Hochschule hin zu einem intern handlungsfähigen ,organisationalen Akteur" (Krücken/Meier 2006) deuten. Dies gilt für die Qualitätsentwicklung, die strategische Planung sowie die Präsidial- und Fakultätsentwicklung. Zudem entstanden in den Bereichen Rektoratsmanagement und Fakultätsmanagement, aber auch im Bereich Controlling/Planung besonders viele Stellen bzw. organisatorische Einheiten. Den stärksten organisationalen Zuwachs hat der Bereich "Studierendenservice und -verwaltung“ erfahren, was vermutlich mit der Umstellung auf Bachelor- und Master-Studiengänge, der Einführung von Studierendenbeiträgen sowie der Entwicklung neuer Beratungsangebote zusammenhängt.

Unterstützt werden diese Aussagen der Kanzler durch die Ergebnisse einer quantitativen Inhaltsanalyse von Stellenanzeigen in der Wochenzeitung „Die Zeit“. Auch hier zeigt sich, dass es einen starken Anstieg an Stellenanzeigen für VollzeitPositionen im Bereich des administrativen Hochschulmanagements gab: Während im Jahr 200373 Stellenanzeigen zu verzeichnen sind, waren es im Jahr 2006 mit 152 mehr als doppelt so viele. Nicht zuletzt die Etablierung einer eigenen Sparte „Hochschulverwaltung“ im Anzeigenteil von „Die Zeit“ weist auf eine zumindest im 
quantitativen Sinne gestiegene Bedeutung dieser Tätigkeitsfelder hin.

Doch was sind Charakteristika dieser neuen Tätigkeitsfelder im mittleren Hochschulmanagement von Hochschuladministrationen? Welche beruflichen Werdegänge, Selbstverständnisse und Qualifikationsanforderungen lassen sich für diesen Berufsbereich konstatieren?

\subsection{WERDEGANG UND ORGANISA- TIONALE VERORTUNG}

Neben dem Kanzler als Kopf der Hochschulverwaltung stellt die Leitung von Dezernaten oder Referaten die Führungsebene der Hochschulverwaltung dar. Diese waren in der Vergangenheit oft mit Mitarbeiterinnen oder Mitarbeitern besetzt, die einen juristischen Ausbildungshintergrund hatten. Dieses war sicherlich im Hinblick auf die Aufgabenstellungen der Hochschulverwaltung zweckdienlich, da die Aufgabe der leitenden Hochschuladministratoren häufig in der Umsetzung von rechtlichen Vorgaben und/oder der Ausführung von Verwaltungsakten lag. Somit war aufgrund des ähnlichen Tätigkeitszuschnitts auch ein Quereinstieg über vorhergehende (Führungs-)Positionen in anderen Verwaltungseinrichtungen oder Organisationen des öffentlichen Sektors möglich und nicht unüblich.

Im Kontext der Reorganisation der Hochschulverwaltungen, insbesondere durch das Hinzukommen weiterer Aufgaben und Servicebereiche, was sich in der Etablierung neuer Organisationseinheiten niederschlägt, sind deutliche Anzeichen einer zunehmenden Heterogenität der fachlichen Hintergründe von leitenden Mitarbeitern im administrativen Management von deutschen Hochschulen festzustellen. Ergebnissen einer deutschlandweiten Befragung von leitenden Mitarbeitern in unterschiedlichen Funktionalbereichen des administrativen Hochschulmanagements zufolge verfügen die meisten von ihnen über Studienabschlüsse im Bereich der Geistes- und Sozialwissenschaften. Zudem scheint es mit zunehmender Nähe zu den Kernaktivitäten Forschung und Lehre auf sehr gute Kenntnisse der Forschungszusammenhänge in der jeweiligen Disziplin anzukommen. So verfügen Mitarbeiter und Geschäftsführer im Bereich des Fakultätsmanagements zumeist über einen Studienabschluss in einer Disziplin ihrer Fakultät bzw. dezentralen Organisa- tionseinheit der Hochschule (Leichsenring 2007). Beides zeigt, dass im hochschulbezogenen Management vor dem Hintergrund organisationaler Transformationsprozesse Tätigkeiten entstanden sind, deren Tätigkeitsprofil nicht ausschließlich auf Regulierungs- und Beschlussumsetzung fokussiert. Damit korrespondiert die abnehmende Bedeutung eines rechtswissenschaftlichen Studienhintergrundes. Ähnliches konnten wir bei unserer Kanzlerbefragung feststellen. Sie zeigt, dass der Studienhintergrund der Hochschulkanzler sich zunehmend wegbewegt von der Rechtswissenschaft als Leitdisziplin und einer Pluralisierung Platz macht, die bei besonderen Zuwächsen in den Wirtschaftswissenschaften im Prinzip sämtliche Fächer von den Ingenieur- und Naturwissenschaften bis zu den Sozial- und Geisteswissenschaften umfasst.

Interessanterweise finden wir für Deutschland trotz der schwindenden Bedeutung öffentlich-rechtlicher Kompetenzen nur geringe Hinweise für eine Hinwendung zu Mitarbeiterinnen und Mitarbeitern, die in privatwirtschaftlichen Kontexten sozialisiert worden sind. Während insbesondere in Großbritannien der Aus- und Aufbau von Hochschulmanagementstrukturen eine intensivierte Rekrutierung von spezialisierten Managementmitarbeitern aus der Privatwirtschaft bedeutete (Neocosmos 2009), ist der Werdegang von leitenden Mitarbeiterinnen und Mitarbeitern im Hochschulmanagement in Deutschland durch eine starke Affinität zum Hochschul- und Wissenschaftssektor geprägt. So verfügen 29,6\% der 492 Teilnehmerinnen und Teilnehmer unserer Befragung (vgl. Fußnote 1) über eine Promotion. Darüber hinaus hat weit über die Hälfte der Befragten vor dem Antritt ihrer jetzigen Stelle Forschungserfahrung als Wissenschaftler oder Wissenschaftlerin gesammelt. Damit scheinen die sich neu entwickelnden Tätigkeitsfelder im Hochschulmanagement insbesondere auch für Grenzgänger aus der Wissenschaft interessant geworden zu sein, die mit einer Tätigkeit am Schnittfeld von Wissenschaft und Administration eine eigenständige Berufsperspektive verbinden. Insbesondere Mitarbeiter in den sogenannten neuen Hochschulprofessionen (Klumpp/Teichler 2005) gehören im Hinblick auf ihr Tätigkeitsfeld und ihre organisatorische Verortung zumeist weder zur klassischen Routineverwaltung noch zum Lehr- und
Forschungspersonal der Universität. Sie agieren in einem Spannungsfeld. Die Etablierung von neuen Tätigkeitsfeldern im Hochschulmanagement impliziert somit vor allem auch eine Hybridisierung beruflicher Rollen und Tätigkeitsfelder. Angesichts dieser Entwicklungen stellt sich die Frage nach der langfristigen Etablierung von Tätigkeitsfeldern zwischen Wissenschaft und herkömmlicher Hochschuladministration und beruflichen Werdegängen im Sinne eines „dritten Weges“ (vgl. dazu auch Torka/Knie in diesem Heft).

\subsection{ARBEITSPROFIL UND BERUF- LICHES SELBSTVERSTÄNDNIS}

Unsere empirischen Untersuchungen zum Berufs- und Tätigkeitsfeld im administrativen Hochschulmanagement machen deutlich, dass dieses Feld sich nicht durch autonomes Entscheiden auszeichnet, sondern vor allem durch intensive Beratung und Koordination der unterschiedlichen Interessengruppen an Hochschulen geprägt ist (vgl. Leichsenring 2007; Adamczak et al. 2007; Klumpp/Teichler 2005). Insofern Hochschulentwicklung mehr denn je auch auf einen spezialisierten Apparat an Instrumenten und wissenschaftliche Ergebnisse zugreift, erlangt die Expertise von spezialisierten Mitarbeitern in den Funktionalbereichen eine wichtigere Rolle. Angesichts steigender Komplexität bei den Instrumentarien der Hochschulsteuerung und einer Pluralisierung der Akteurskonstellationen im Hochschulbereich sind Hochschul- und Fakultätsleitungen zunehmend auf das spezialisierte Wissen ihrer Mitarbeiter und Mitarbeiterinnen in den jeweiligen Funktionalbereichen angewiesen.

Damit wird deutlich, dass das zentrale Moment des Arbeitsprofils im administrativen Hochschulmanagement nicht unternehmerische Umsetzung oder professionelle Autonomie ist, sondern bereichspezifisches Wissen und Organisationserfahrung. Die Einspeisung von spezialisierten Managementkenntnissen und Wissen für den jeweiligen Tätigkeitsbereich baut auf der Anpassungsfähigkeit und Sensibilität der Mitarbeiter im Hochschulmanagement sowie deren Beratungs- und Verhandlungskompetenz auf. So verwundert es auch nicht, dass in unserer Umfrage unter Leiterinnen und Leitern von Funktionalbereichen vor allem „soft-skills“ wie Teamfähigkeit, Verhandlungs- und Beratungs- 
geschick als wichtigste Kompetenzen für die eigene Tätigkeit genannt wurden. Das ist sicherlich auf das Tätigkeitsprofil dieser Gruppe zurückzuführen, welches sich durch eine intensive Zusammenarbeit mit vielen verschiedenen Personengruppen vor allem innerhalb der Hochschule auszeichnet.

Die starke Bezogenheit auf hochschulinterne Prozesse und Personen zeigt sich zudem auch in dem erfragten Selbstverständnis: Dieses wurde häufig als „Servicedienstleister" und als „Berater für meinen Aufgabenbereich innerhalb der Hochschule“ umschrieben. In einem Interview mit einem leitenden Mitarbeiter im Bereich Rektoratsmanagement wurde diesbezüglich angeführt: „Ich denke vor allem aus Sicht der Organisation: Ich sehe es als meine zentrale Aufgabe, verschiedene Organisationsinteressen zusammenzubringen. " Diese Selbsteinschätzung und Orientierung sind insofern bemerkenswert, als sie sich diametral zu den selbst gewählten Referenzpunkten von Professorinnen und Professoren verhalten. In Langzeitstudien zu akademischen Professionen zeigte sich, dass Professorinnen und Professoren sich am stärksten ihrer Disziplin verpflichtet fühlen, dann dem Fachbereich und erst als Letztes der eigenen Hochschule (RIHE 2009). Man könnte vermuten, dass durch die starke Identifikation mit der eigenen Organisation die im administrativen Hochschulmanagement Tätigen als „Promotoren“ (Hauschildt/Gemünden 1999) des Wandels der ehemals lose gekoppelten Organisation Hochschule hin zu einem organisationalen Akteur fungieren, welcher sich durch eine eigene Identität sowie hierauf bezogene Ziele und Strategien auszeichnet.

\subsection{QUALIFIKATION UND VERNETZUNG}

Obgleich der zentrale Referenzpunkt der im administrativen Hochschulmanagement Tätigen die eigene Hochschule ist, gewinnen professionelle Netzwerke im Hochschulmanagement zunehmend an Bedeutung. So ist es in den letzten Jahren in Deutschland zur Institutionalisierung und Erweiterung deutschlandweiter Dachverbände und zahlreicher Netzwerke bzw. Portale in spezifischen Tätigkeitsfeldern des Hochschulmanagements gekommen. Beispiele sind das Career Service Netzwerk (www.csnd.de), der Verband der Alumni-Organisationen oder das Netzwerk der Forschungsreferenten (www.forschungsreferenten.de).
Diese Netzwerke weisen nur wenige Gemeinsamkeiten mit traditionellen Berufsvereinigungen im Sinne der Professionssoziologie auf. ${ }^{5}$ Dennoch übernehmen sie wichtige Funktionen im neuen Berufsfeld „Hochschulmanagement“. So schaffen sie durch das Angebot von funktionalbereichsspezifischen Seminaren und Fortbildungen Grundlagen für eine gemeinsame Wissensbasis, für die der Austausch über Arbeits- und Organisationspraktiken sowie Problemwahrnehmungen von zentraler Bedeutung ist. Angesichts heterogener Umwelterwartungen und -wahrnehmungen, mit denen Mitarbeiter im administrativen Hochschulmanagement vor allem aufgrund ihrer Verortung zwischen Verwaltung und Wissenschaft konfrontiert sind, sowie eines Mangels an eindeutigen Problemlösungstechniken machen es diese bereichsspezifischen Netzwerke möglich, praktikable „Coping-Strategien“ zu finden. Darüber hinaus dienen sie dazu, eine Art Rückversicherung für das eigene Handeln zu liefern.

Soziologisch betrachtet findet in derartigen Netzwerken eine Selbst- und Fremdreflexion statt, die man mit Strang/Meyer (2009, S. 140ff.) als Theoretisieren bezeichnen kann. „Theoretisieren“ bedeutet zwar keine wissenschaftliche Reflexion, jedoch die Fähigkeit, eigene Erfahrungen durch Abstraktion in einen breiteren Kontext zu stellen und somit für andere verfügbar zu machen. Dies ist die Grundlage für Sinngebungs- und Verständigungsprozesse im Feld. Im Gegensatz zur Orientierung am singulären Fall erlaubt Abstraktion zudem die Diffusion von übertragbaren Handlungsmodellen. Eine weitere wichtige Funktion der Netzwerke ist neben der Selbst- und Fremdreflexion die Suche nach einem gemeinsamen beruflichen Selbstverständnis. Dieses Suchen ist umso wichtiger, als sich Mitarbeiterinnen und Mitarbeiter insbesondere in den neuen Tätigkeitsbereichen ja gerade nicht durch eine ähnliche berufliche Sozialisation auszeichnen und ihr Tätigkeitsprofil sowie ihre Verortung innerhalb der Hochschule Gegenstand ständiger Aushandlungen sind. Da sich die eigene berufliche Identität nicht nur aus der personalen Identität, sondern immer auch aus der sozialen Identität konstituiert und diese dann angesprochen wird, "wenn sich die Person vorrangig als Mitglied einer bestimmten Gruppe und weniger als einzigartiges Individuum betrachtet" (Blanz 1998, S. 2), kann somit den Netzwerken auch eine wichtige sozialpsychologische Funktion zugesprochen werden. So kann die Institutionalisierung solcher beruflichen und professionellen Netzwerke langfristig auch ein wichtiges Element für die Institutionalisierung der Berufs- und Tätigkeitsfelder im Hochschulmanagement sein.

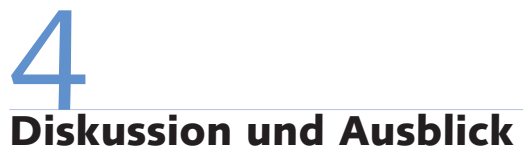

Auf der Basis unserer Ausführungen wird deutlich, dass aufgrund der fehlenden gemeinsamen beruflichen Sozialisation, des fehlenden gemeinsamen Wissenskanons sowie der im Vergleich zu traditionellen Berufsverbänden eingeschränkten Befugnisse und Kompetenzen der Netzwerke nicht von einer Profession Hochschulmanagement im klassischen professionssoziologischen Sinn gesprochen werden kann.

Dennoch, und hierin spiegeln sich auch neuere professionssoziologische Entwicklungen wider, kann den im administrativen Hochschulmanagement Tätigen durchaus Professionalität zugesprochen werden, und zwar jenseits der alltagssprachlichen Gleichsetzung mit kompetentem Handeln. So wird in der neueren Professionssoziologie auch die organisationale Verortung, die traditionell eher im Gegensatz zur Verortung innerhalb einer Profession gesehen wurde, als positives Definitionsmerkmal berücksichtigt.

Julia Evetts (2008) bietet für das Tätigkeitsfeld Hochschulmanagement, das hier zur Diskussion steht, eine wichtige Unterscheidung zweier distinkter Formen von Professionalismus an. Sie differenziert idealtypisch zwischen einer an traditionellen Professionskonzepten angelehnten „beruflichen Professionalität" sowie einer „organisationalen Professionalität“ (Evetts 2008). Letztere zeichne sich dadurch aus, dass es im Gegensatz zur beruflichen Professionalität keine homogene berufliche Sozialisation gibt, kein spezifisches Professionsethos und keine kollegiale Autorität im Zusammenhang mit einer starken Orientierung an den eigenen Peers. Statt-

5 Die Funktionen eines Berufsverbandes werden zumeist mit Kanonisierung des Wissens sowie einer Kontrolle des Zugangs und der Tätigkeitsausübung verbunden (vgl. z.B. Parsons 1968; Wilensky 1964). 
dessen sei eine rational-legalistische Form der Autorität vorherrschend, Arbeitsprozeduren seien stärker durch organisationale Vorgaben bestimmt. Zudem fänden sich die Mitarbeiter und Mitarbeiterinnen in hierarchische Strukturen von Autorität und Entscheidungsfindung innerhalb der eigenen Organisation eingebettet. Dennoch seien auch hier Prozesse der Spezialisierung und Verwissenschaftlichung von Arbeitsfeldern sichtbar.

Übertragen auf unseren Fall bedeutet dies, Indikatoren für Professionalisierungsprozesse vor allem in der Institutionalisierung von spezialisierten Aus- und Weiterbildungsstudiengängen, beruflichen und professionellen Netzwerken sowie in der Herausbildung eines spezifischen Berufsbildes zu sehen, während im Unterschied zur „beruflichen Professionalität" weder die Autonomie gegenüber der Organisation noch die Regulierung des Zugangs zum Feld gegeben sind. Hieran schließen zahlreiche Fragen an, die sowohl für das wissenschaftliche Verständnis als auch für die Alltagspraxis der in diesem Arbeitsfeld Tätigen von hoher Bedeutung sind.

(1) Es stellt sich die Frage, wie weit diese Professionalisierungsprozesse reichen und welche Rückwirkungen auf die Organisation hieraus resultieren. In Deutschland sind gegenwärtig eher lose geknüpfte Netzwerke, die dem Informations- und Wissensaustausch sowie der Selbst- und Fremdreflexion dienen, zu beobachten. Hier bestehen zum Teil erhebliche Unterschiede zu den USA, wie ein Blick auf die im Bereich Forschungs- und Technologietransfer tätigen Mitarbeiterinnen und Mitarbeiter („Transfermanager") und deren Vernetzung zeigt. So gibt es in den USA eine über die Landesgrenzen anerkann- te Berufsvereinigung, die Association of University Technology Managers (AUTM), die spezifische Qualifikationsprogramme anbietet, über spezialisierte Arbeitsgruppen wie auch über vielfältige Kanäle der Wissensdiffusion und Standardisierung von Praktiken verfügt. Eine Berufsvereinigung wie AUTM fungiert als Katalysator für Strukturangleichungen zwischen Universitäten, und zwar in einem wichtigen Bereich der sich neuen Herausforderungen stellenden und strategisch positionierenden Gesamtorganisation „Universität“.

Gegenwärtig lassen sich für Deutschland derartige Entwicklungen nicht konstatieren, weder im Bereich des Technologietransfers noch in den anderen von uns untersuchten Feldern. Es ist eine offene und spannende Frage, ob damit auch für Deutschland zukünftig relevante Entwicklungen vorweg genommen werden, oder ob derart weitreichende Professionalisierungsprozesse aufgrund der Unterschiedlichkeit der Hochschulsysteme und der darin eingebetteten Hochschulen auch zukünftig nicht anzutreffen sein werden.

(2) Es besteht ein gewisses Spannungsverhältnis zwischen dem Anspruch der im administrativen Hochschulmanagement Tätigen, die Interessen der Gesamtorganisation zu vertreten, und der deutlichen Fokussierung auf den jeweils spezifischen Arbeitsbereich. Wie in Abschnitt 3.2. ausgeführt, besteht eine hohe Loyalität gegenüber der Gesamtorganisation, die diesen Personenkreis als Promotoren des Wandels der Universität zu einem einheitlich agierenden organisationalen Akteur erscheinen lassen. Jedoch ist die starke Identifikation mit dem eigenen Arbeitsbereich nicht unproblematisch, da so wiederum Partikularinteressen im Vordergrund stehen:
Wer stimmt die durchaus berechtigten Anliegen der Controller, Internationalisierer, IT-Vernetzer, Gleichstellungsbeauftragten, Qualitätssicherer und Öffentlichkeitsarbeiter aufeinander $\mathrm{ab}$ ? Was sind geeignete Strukturen, damit das Gesamtinteresse der Organisation gewahrt bleibt? Eine organisationsinterne Möglichkeit besteht vermutlich darin, dass die Aufgabenbereiche der Mitarbeiterinnen und Mitarbeiter des Öfteren wechseln bzw. neu zugeschnitten werden, auch wenn hiermit vermutlich Reibungsverluste verbunden sind. Organisationsübergreifend ist vor allem an Ausund Weiterbildungsangebote zu denken, die sich auf das Hochschulmanagement insgesamt beziehen und die ein sinnvolles Gegengewicht zur Überidentifikation mit dem jeweils spezifischen Aufgabenbereich schaffen.

(3) Aus unserer Sicht ist es eine empirisch offene Frage, inwiefern sich diese neuen Tätigkeitsbereiche im administrativen Hochschulmanagement nachhaltig etablieren und Aufstiegs- und Weiterentwicklungsperspektiven bieten. Dies betrifft insbesondere die Frage eines „dritten Karrierewegs" zwischen Wissenschaft und Verwaltung. Dafür sprechen vor allem die neuen Herausforderungen an die Hochschulen sowie die Qualifikationsprofile derjenigen, die in diesen Bereichen tätig sind. Dagegen sprechen jedoch die traditionellen universitären Karrierepfade, deren Ziele entweder in der Professur oder in einer leitenden Verwaltungsposition bestehen. Diese Pfade sind über Jahrhunderte entstanden. Sie lassen sich kaum kurzfristig um einen neuen Pfad, der einer eigenen Logik und Rekrutierungspraxis folgt, ergänzen. 
Adamczak, W./Debusmann, A./Merkator, N. (2007): Traumberuf ForschungsreferentIn?, Werkstadtbericht 68, Kassel

Blanz, M. (1998): Wahrnehmung von Personen als Gruppenmitglieder: Untersuchungen zur Salienz sozialer Kategorien, Texte zur Sozialpsychologie Bd. 4, Münster

Chandler, A. (1977): The Visible Hand: The Managerial Revolution in American Business, Cambridge, Mass.

Clark, B.R. (1998): Creating Entrepreneurial Universities. Organizational Pathways of Transformation, Surry

Clark, B.R. (2004): Sustaining Change in Universities. Continuities in Case Studies and Concepts, Maidenheed

Evetts, J. (2008): Professionalität durch Management? Neue Erscheinungsformen von Professionalität und ihre Auswirkungen auf professionelles Handeln. Ein Nachtrag zum ZSR-Schwerpunktheft 3/2007,

in: Zeitschrift für Sozialreform 1, S. 97-106

Hauschildt, J./Gemünden, H.G. (Hrsg.) (1999): Promotoren. Champions der Innovation, Wiesbaden

Hüther, O. (2009): Von der Kollegialität zur Hierarchie? Eine Analyse des New Managerialism in den Landeshochschulgesetzen, unveröffentlichte Dissertation, Universität Hamburg, Fachbereich Sozialwissenschaft

Klumpp, M./Teichler, U. (2005): Experten für das Hochschulsystem. Hochschulprofessionen zwischen Wissenschaft und Administration, in: Kehm, B./Mayer, E./Teichler, U. (Hrsg.): Hochschulprofessionen zwischen Wissenschaft und Administration. Hochschule Innovativ 4, S. 2-3

Kosmützky, A. (2010): Von der organisierten Institution zur Institutionalisierung von Organisation? Eine Untersuchung der (Hochschul-) Leitbilder von Universitäten, unveröffentlichte Dissertation, Universität Bielefeld, Fakultät für Soziologie

Krücken, G. (2003): Mission Impossible? Institutional Barriers to the Diffusion of the 'Third Academic Mission' at German Universities, in: International Journal of Technology Management 1/2, S. 18-33

Krücken, G./Blümel, A./Kloke, K. (2009): Towards Organizational Actorhood of Universities: Occupational and Organizational Change within German University Administration, FÖV-Discussion Papers 48, Speyer
Krücken, G./Meier, F. (2006): Turning the University into an Organizational Actor, in: Drori, G./Meyer, J./Hwang, H. (Hrsg.): Globalization and Organization, Oxford, S. 241-257

Lange, S./Schimank, U. (2007): Zwischen Konvergenz und Pfadabhängigkeit: New Public Management in den Hochschulsystemen fünf ausgewählter OECD-Länder, in: Holzinger, K./Joergens, H./Knill, C. (Hrsg.): Transfer, Diffusion und Konvergenz von Politiken, Sonderheft 38 der Politischen Vierteljahresschrift, S. 522-548

Leichsenring, H. (2007): Die Professionalisierung des Fakultätsmanagements - Bericht zur Befragung 2006, Gütersloh, Centrum für Hochschulentwicklung

Luckwald, J. von (2008): Stellenwert der Career Service-Arbeit an deutschen Universitäten, in: Seeber, G. (Hrsg.): Forschungsfelder der Wirtschaftsdidaktik, Schwalbach, S. 192-200

Mintzberg, H. (1979): The Structuring of Organizations, New Jersey Neocosmos, S. (2009): Professionalisation: The British Experience. Vortrag beim 6. Kolloquium Hochschul- und Wissenschaftsmanagement am 5.3. an der FH Osnabrück

Nickel, S. (2007): Partizipatives Management von Universitäten. Zielvereinbarungen, Leitungsstrukturen, staatliche Steuerung, Mering Parsons, T. (1968): Professions, in: International Encyclopedia of the Social Sciences 12, S. 536-547

Polanyi, M. (1966): The Tacit Dimension, New York

Research Institute for Higher Education (RIHE) (Hrsg.) (2009): The Changing Academic Profession in International Comparative and Quantitative Perspectives, Hiroshima University, Hiroshima

Schelsky, H. (1963): Einsamkeit und Freiheit. Idee und Gestalt der deutschen Universität und ihrer Reformen, Düsseldorf

Strang, D./Meyer, J.W. (2009): Institutional Conditions for Diffusion, in: Krücken, G./Drori, G. (Hrsg.): World Society. The Writings of John Meyer, Oxford, S. 136-155

Wilensky, H. (1964): The Professionalization of Everyone?, in: American Journal of Sociology 2, S. 137-158

Ziegele, F. (2005): Umsetzung der neuen Steuerungsmodelle (NSM) im Hochschulrecht, in: Fisch, R./Koch S. (Hrsg.): Neue Steuerung von Bildung und Wissenschaft, Schule - Hochschule - Forschung, Bonn, S. 107-121 